\title{
RIGIDEZ DO PAPELÃO ONDULADO: COMPARAÇÃO ENTRE RESULTADOS EXPERIMENTAIS E OS OBTIDOS POR CÁLCULO ANALÍTICO ${ }^{1}$
}

\author{
PAUlo G. MAGALHÃES ${ }^{2}$, PAUlO R. DE A. FIGUEIREDO ${ }^{3}$, FRANCO G. DEDINI ${ }^{4}$
}

\begin{abstract}
RESUMO: Embalagens de papelão ondulado para produtos hortícolas têm como função principal a proteção do produto. $\mathrm{O}$ dimensionamento de uma embalagem de papelão requer o conhecimento da rigidez à flexão, que depende dos módulos de elasticidade dos elementos que o constituem. Este trabalho teve por objetivo calcular, a partir da caracterização física do papelão em laboratório, o módulo de elasticidade por diferentes métodos, comparando os resultados com os valores obtidos experimentalmente. Dez corpos de prova de cada um dos papéis selecionados para este estudo foram testados na direção de fabricação e na direção transversal. A resistência à tração dos papéis, capa e miolo, utilizada para calcular a rigidez, foi determinada em máquina universal de ensaios. Para a obtenção da rigidez à flexão, foi realizado o teste de quatro pontos. Foi observada expressiva variação entre os métodos pelos quais se obtêm os módulos de elasticidade que reflete nos valores de rigidez da estrutura. Os valores de rigidez obtidos experimentalmente foram sempre superiores aos valores obtidos por cálculos analíticos. Essa diferença pode ser atribuída a dois fatores conjugados: o processo de fabricação que confere maior rigidez do que os componentes isoladamente, e o outro componente é a adição de camada adesiva que não é levada em consideração nos cálculos analíticos.
\end{abstract}

PALAVRAS-CHAVE: embalagem, módulo de elasticidade, rigidez à flexão.

\section{BENDING STIFFNESS EVALUATION OF CARDBOARD: COMPARISON BETWEEN EXPERIMENTAL AND ANALYTICALLY RESULTS}

\begin{abstract}
Cardboard packing for horticultural products has as main function to protect them. The design of a cardboard packing request the knowledge of the bending stiffens which is depending on the modulus of elasticity. The objective of this work was to calculate the cardboard modulus of elasticity from data obtained in laboratory using physical characterization test, with different methods, and comparing the results with the values obtained experimentally. Ten samples of each cardboard selected for this study were tested in the paper fabrication direction and in its transverse direction. The papers liner and medium resistance to the traction, used to calculate the bending stiffness, was determined in a universal machine test. To obtaining of the bending stiffens the four points test was accomplished. Expressive variations among the methods from which the modulus of elasticity is obtained were observed and that influence the bending stiffness of the structure. The stiffness values obtained experimentally were always greater than the values obtained from analytical method. This difference can be attributed to two factors, the production processes that assurance a larger rigidity than the components separately and the addition of the adhesive layer that is not taken in consideration in the analytic calculations.
\end{abstract}

KEYWORDS: cardboard, young modulus, bending stiffness.

\footnotetext{
${ }^{1}$ Extraído da tese de doutorado desenvolvida na Faculdade de Engenharia Mecânica da UNICAMP, com apoio do CNPq.

${ }^{2}$ Prof. Titular, Faculdade de Engenharia Agrícola, UNICAMP, graziano@agr.unicamp.br

${ }^{3}$ Eng ${ }^{\text {o }}$ Agrícola, Pesquisador do Instituto Agronômico do Paraná - IAPAR e aluno do curso de doutorado da FEM/ UNICAMP.

${ }^{4}$ Professor Livre Docente da Faculdade de Engenharia Mecânica da UNICAMP.

Recebido pelo Conselho Editorial em: 24-8-2004

Aprovado pelo Conselho Editorial em: 13-12-2005
} 


\section{INTRODUÇÃO}

O papelão ondulado, por suas características construtivas, é um material com qualidades únicas, como: facilidade construtiva, custo reduzido em relação a sua capacidade de contenção do produto e resistência mecânica. Essa última característica está diretamente relacionada com a principal função que deve exercer uma embalagem, que é a proteção do produto (PICHLER, 2000).

Para atingir mercados cada vez mais distantes, fornecedores de produtos hortícolas têm de apresentar embalagens que garantam sua integridade, tenham boa aparência, levem em consideração os fatores ambientais e também a prevenção de injúria mecânica causada durante o transporte (CHITARRA, 1990). Assim, no projeto de uma embalagem de papelão, a necessidade de avaliar os níveis de abertura (quantidade, formato e disposição), além da capacidade de carga, utilizando o procedimento de tentativa e erro, torna-se muito caro e moroso. A modelagem do papelão ondulado, para o dimensionamento de uma embalagem, utilizando o método dos elementos finitos, requer o conhecimento das propriedades mecânicas do material e o conhecimento das variações dimensionais existentes em decorrência do processo de fabricação.

Os diferentes níveis de resistência de uma estrutura de papelão estão diretamente relacionados às diferenças de rigidez à flexão dessas estruturas (POMMIER \& POUTIS, 1989). A rigidez à flexão depende dos módulos de elasticidade dos elementos que o constituem: capa, contra-capa e miolo, e também da geometria do miolo, devido aos tipos de ondas.

Dessa forma, este trabalho teve como objetivo realizar a caracterização mecânica (rigidez à flexão e resistência à tração), em laboratório, do papelão de diferentes gramaturas e calcular, a partir desses resultados, o módulo de elasticidade por diferentes métodos (resistência à tração e rigidez Taber), comparando os resultados com os valores obtidos experimentalmente.

\section{MATERIAL E MÉTODOS}

Os testes de determinação das propriedades do papelão foram realizados no laboratório de embalagens do Instituto Tecnológico de Alimentos de Campinas, ITAL. Todos os testes foram conduzidos em condições controladas de temperatura e umidade, mantendo-se a $23{ }^{\circ} \mathrm{C}$ e umidade relativa de $50 \%$.

Nos ensaios para a determinação das propriedades mecânicas, rigidez à flexão pelo método TAPPI e quatro pontos, e a resistência à tração, os componentes utilizados foram: papel-capa fabricado de material virgem, processo kraft, e papéis-miolo fabricados a partir de aparas fornecidos pela Klabin - Fabricante de Papel e Celulose S.A., de Jundiaí - SP. O material utilizado, produzido comercialmente, foi retirado das bobinas antes de entrar no processo de fabricação das estruturas. Para os papéis-capa, foram utilizadas três gramaturas nominais de fabricação: $125 ; 140$ e $200 \mathrm{~g} \mathrm{~m}^{-2}$. As gramaturas nominais de fabricação do papel-miolo reciclado foram 120; $140 \mathrm{e} 170 \mathrm{~g} \mathrm{~m}^{-2}$.

\section{Determinação da rigidez à flexão dos componentes da estrutura do papelão}

Na determinação da rigidez à flexão dos papéis-capa e miolo, utilizou-se da metodologia TAPPI T489 om-92 - "Stiffenss of paper and paperboard" (BAUM \& HABEGER, 1980), e do aparelho Taber - Stiffenss Tester (Figura 1). Esse método determina o momento de flexão necessário para fletir um corpo-de-prova de $38,1 \pm 3 \mathrm{~mm}$ de largura, $15^{\circ}$ da sua linha de centro, com força aplicada a $50 \mathrm{~mm}$ do ponto onde o corpo-de-prova é fixado, sendo os resultados expressos em $\mathrm{gf} \mathrm{cm}$. 


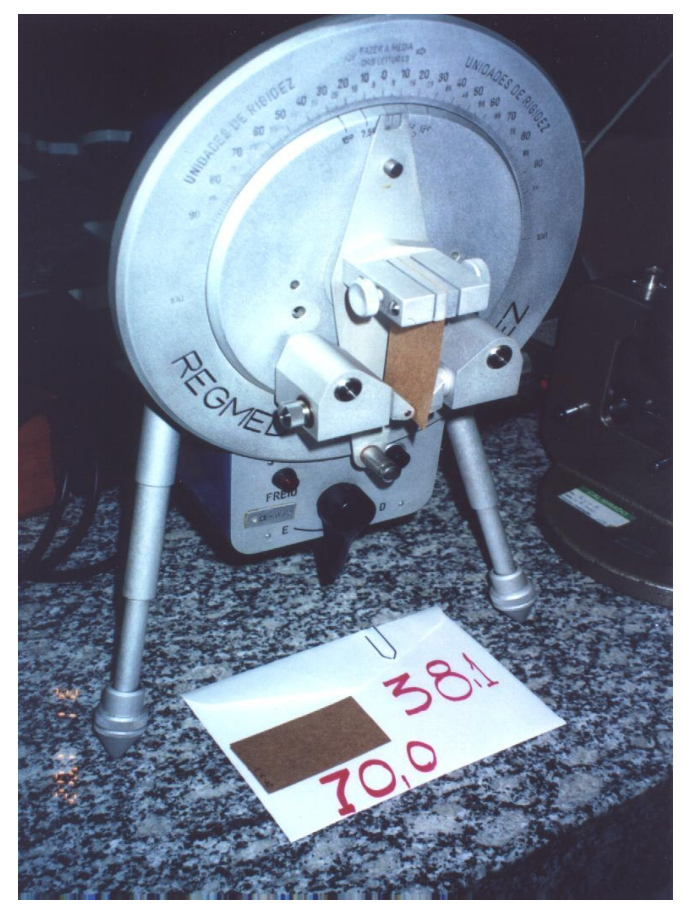

FIGURA 1. Ensaio dos papéis-capa e miolo no aparelho Taber - "Stiffness Tester”.

A rigidez à flexão do material (bending stiffness), definida como sendo o módulo de elasticidade vezes o momento de inércia, por unidade de largura, foi calculada pela eq. (1).

$$
\mathrm{E} I=\frac{\mathrm{FL}^{3}}{3 \mathrm{Y}}
$$

em que,

$\mathrm{Y}$ - deslocamento, mm;

F - força, N;

$\mathrm{L}$ - comprimento do corpo-de-prova, $\mathrm{mm}$;

E - módulo de elasticidade, $\mathrm{N} \mathrm{mm}^{-2}$, e

$\mathrm{I}$ - momento de inércia, $\mathrm{mm}^{3}$.

Como a deflexão do corpo-de-prova é conhecida e igual a $15^{\circ}$, temos $\mathrm{Y} / \mathrm{L}=\operatorname{sen} 15^{\circ}$, portanto:

$$
\mathrm{E} I=\mathrm{F}\left(\frac{\mathrm{L}^{2}}{3}\right) \operatorname{sen} 15^{\circ}
$$

Sendo F L = Rigidez Taber, a eq.(2) pode ser escrita da seguinte forma:

$$
\mathrm{E} I=\operatorname{Rigidez} \operatorname{Taber}\left(\frac{\mathrm{L}}{3}\right) \operatorname{sen} 15^{\circ}
$$

Dez corpos-de-prova de cada um dos papéis selecionados para este estudo foram testados nas direções do papel, identificadas como: DF - direção de fabricação e DT - direção transversal.

\section{Determinação da resistência à tração dos componentes da estrutura do papelão}

A resistência à tração dos papéis-capa e miolo foi determinada em máquina universal de ensaios INSTRON, modelo $5500 \mathrm{R}$, com uso de célula de carga de $500 \mathrm{~N}$. A resistência à tração foi determinada para calcular o módulo de elasticidade e o coeficiente de Poison do material nas direções 
transversal e de fabricação. Os corpos-de-prova foram cortados com auxílio de uma guilhotina com largura-padrão de 15,0 \pm 0,1 mm e comprimento de 250,0 \pm 0,1 mm, sendo submetidos a esforço de tração nas direções transversal e longitudinal, e a velocidade constante até sua ruptura. A resistência à tração foi calculada, para cada direção, separadamente, utilizando-se da eq. (4).

$$
\mathrm{R}=\frac{\mathrm{F}}{\mathrm{L}}
$$

em que,

$\mathrm{R}$ - resistência à tração, por unidade de largura, em uma das direções, $\mathrm{kN}$ m;

$\mathrm{F}$ - força de ruptura média, $\mathrm{N}$, e

L - largura do corpo-de-prova, $\mathrm{m}$.

A percentagem de alongamento do corpo-de-prova foi determinada utilizando-se da eq. (5):

$$
\% \mathrm{~A}=\left(\frac{\mathrm{df}-\mathrm{di}}{\mathrm{di}}\right) 100
$$

em que,

df - distância entre as garras no instante de ruptura, m, e

di - distância entre as garras no início do ensaio, m.

\section{Composição das estruturas de papelão ondulado utilizadas}

Nos ensaios, foi avaliado o comportamento das estruturas de papelão ondulado de onda $\mathrm{C}$ e $\mathrm{BC}$, Figura 2.

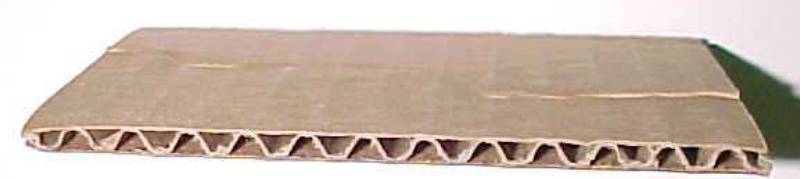

(a)

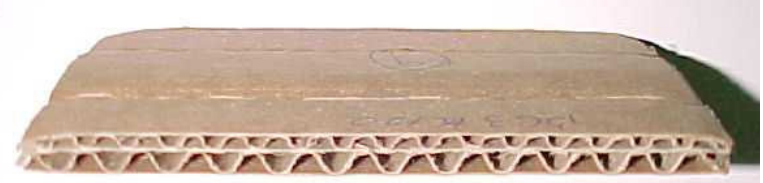

(b)

FIGURA 2. Estrutura de papelão ondulado de onda C (a) e onda BC, onda B na parte superior da estrutura e onda $\mathrm{C}$ na parte inferior (b).

As estruturas de papelão ondulado utilizadas foram retiradas da linha de produção de embalagens antes de haver o corte para confecção das embalagens. As composições das estruturas avaliadas apresentam duas formas de composição para as estruturas com onda $C$ (Tabela 1) e duas formas de composição para as estruturas com onda BC (Tabela 2).

TABELA 1. Composição das estruturas de onda $\mathrm{C}$, de acordo com a gramatura nominal $\mathrm{g} \mathrm{m}^{-2}$.

\begin{tabular}{cccc}
\hline Nomenclatura & Capa Externa Kraft & Miolo Reciclado & Capa Interna Kraft \\
\hline CK45 & 125 & 120 & 125 \\
CK58 & 140 & 170 & 140 \\
\hline
\end{tabular}


TABELA 2. Composição das estruturas de onda BC, de acordo com a gramatura nominal $\mathrm{g} \mathrm{m}^{-2}$.

\begin{tabular}{cccccc}
\hline Nomenclatura & $\begin{array}{c}\text { Capa Externa } \\
\text { Kraft }\end{array}$ & $\begin{array}{c}\text { Miolo Onda B } \\
\text { Reciclado }\end{array}$ & $\begin{array}{c}\text { Miolo Plano } \\
\text { Reciclado }\end{array}$ & $\begin{array}{c}\text { Miolo Onda C } \\
\text { Reciclado }\end{array}$ & $\begin{array}{c}\text { Capa Interna } \\
\text { Kraft }\end{array}$ \\
\hline BCK85 & 140 & 140 & 140 & 140 & 140 \\
BCK120 & 200 & 170 & 170 & 170 & 200 \\
\hline
\end{tabular}

\section{Determinação da rigidez à flexão da estrutura do papelão}

Dentre os testes existentes, o mais utilizado para a obtenção da rigidez à flexão principal é o teste dos quatro pontos, em virtude de a força aplicada não causar tensões de cisalhamento na placa entre os pontos de aplicação. Esse teste foi realizado com um aparelho da Lorentzen \& Wettre (MOURAD et al., 1999), Figura 3.

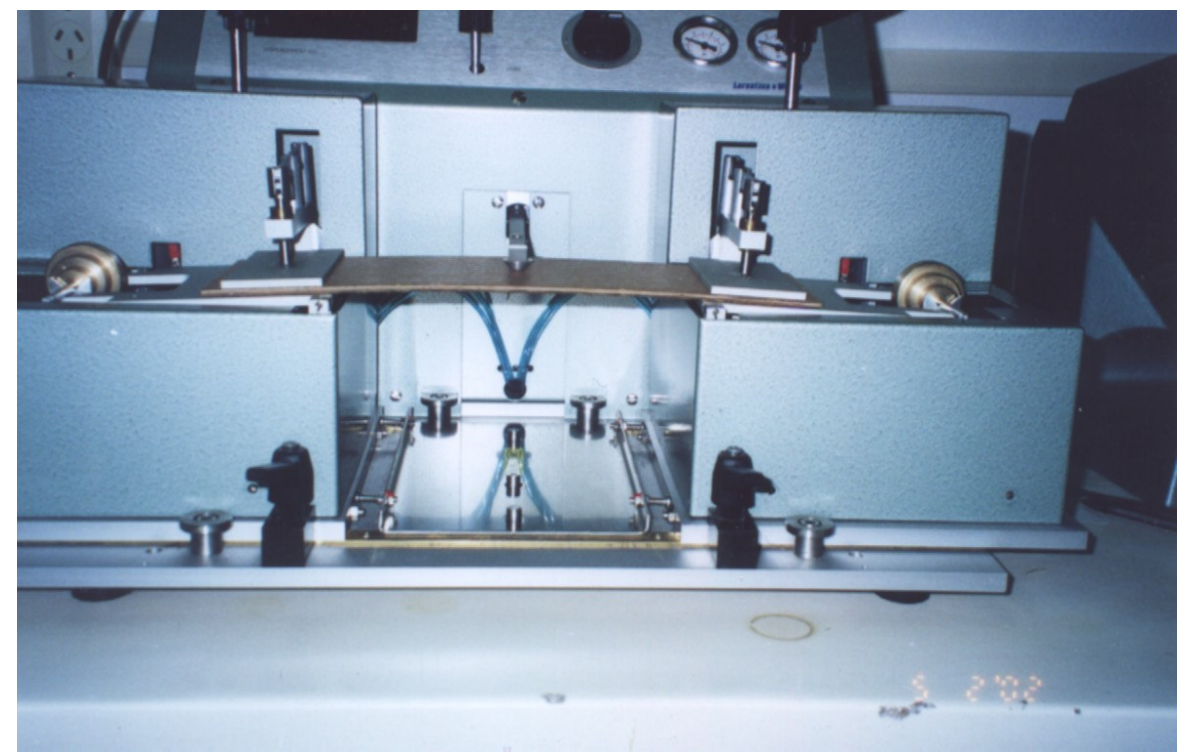

FIGURA 3. Ensaio de rigidez à flexão da estrutura de papelão ondulado em aparelho de quatro pontos.

A rigidez é obtida pela eq. (6).

$$
\mathrm{D}_{11} \text { e } \mathrm{D}_{22}=\left(\frac{\mathrm{P}}{2} \mathrm{~L}_{1} \mathrm{~L}^{2}\right) /(8 \mathrm{Y} \mathrm{w})
$$

em que,

$\mathrm{D}_{11}$ e $\mathrm{D}_{22}$ - rigidez à flexão nas direções perpendicular e na direção da onda, $\mathrm{N}$ m;

$\mathrm{P} / 2$ - carga aplicada em cada extremidade, $\mathrm{N}$;

$\mathrm{L}_{1}$ - distância do apoio até a extremidade onde é aplicada a carga, m;

L - distância entre os apoios, m;

$\mathrm{Y}$ - deslocamento devido à flexão, $\mathrm{m}, \mathrm{e}$

$\mathrm{w}$ - largura do corpo de prova, $\mathrm{m}$.

Para que se possa analisar o papelão ondulado como um conjunto de placas ortotrópicas homogêneas equivalentes, sobrepostas de tal modo que formem uma única estrutura, é necessário que se obtenham as propriedades mecânicas equivalentes de cada camada. O cálculo da rigidez de cada elemento constituinte do papelão ondulado, necessário na análise estrutural da placa, foi obtido com o procedimento de cálculo proposto por CARLSSON et al. (1985). 
Para as estruturas de parede simples, com uma ondulação central, miolo e duas placas laterais de capas, o cálculo da rigidez equivalente é como se segue:

$$
\begin{aligned}
& \mathrm{D}_{11}=\frac{\mathrm{E}_{\mathrm{x}}^{\mathrm{c}}}{1-v_{\mathrm{xy}}^{\mathrm{c}} v_{\mathrm{yx}}^{\mathrm{c}}} \mathrm{I}^{\mathrm{c}} \\
& \mathrm{D}_{22}=\frac{\mathrm{E}_{\mathrm{y}}^{\mathrm{c}}}{1-v_{\mathrm{xy}}^{\mathrm{c}} v_{\mathrm{yx}}^{\mathrm{c}}} \mathrm{I}^{\mathrm{c}}+\frac{\mathrm{E}_{\mathrm{y}}^{\mathrm{m}}}{1-v_{\mathrm{xy}}^{\mathrm{m}} v_{\mathrm{yx}}^{\mathrm{m}}} \mathrm{I}^{\mathrm{m}} \\
& \mathrm{D}_{12}=\frac{v_{\mathrm{xy}}^{\mathrm{c}} \mathrm{E}_{\mathrm{y}}^{\mathrm{c}}}{1-v_{\mathrm{xy}}^{\mathrm{c}} v_{\mathrm{yx}}^{\mathrm{c}}} \mathrm{I}^{\mathrm{c}} \\
& D_{x y}=G_{x y}^{c} t^{c}+G_{x y}^{m} I^{m} \\
& \mathrm{I}^{\mathrm{m}}=\frac{1}{9 \pi}\left\{\left[3+\left(\frac{\mathrm{t}^{\mathrm{m}}}{\mathrm{t}^{\mathrm{c}}}\right)^{2}\right]+\frac{2 \eta \eta_{\mathrm{c}}}{2 \mathrm{p}}\left[1+3\left(\frac{\mathrm{t}^{\mathrm{m}}}{\mathrm{h}_{\mathrm{c}}}\right)^{2}\right]\right\} \mathrm{h}_{\mathrm{c}}{ }^{2} \mathrm{t}^{\mathrm{m}} \\
& I^{c}=\left\{\frac{1}{2}-\frac{t^{c}}{t}+\frac{2}{3}\left(\frac{t^{c}}{t}\right)^{2}\right\} t^{2} t^{c} \\
& \eta=\left\{1+\left(\frac{\mathrm{p} / 2+\mathrm{t}^{\mathrm{m}}}{\mathrm{a} \pi}\right)^{2}\right\}^{1 / 2}
\end{aligned}
$$

em que,

$E_{x, y}^{c}$ - módulo de elasticidade da capa nas direções $\mathrm{x}$ ou y;

$\mathrm{E}_{\mathrm{x}, \mathrm{y}}^{\mathrm{m}}$ - módulo de elasticidade do miolo nas direções $\mathrm{x}$ ou y;

$\mathrm{G}_{\mathrm{xy}}^{\mathrm{c}}$ - módulo de torção da capa no plano xy;

$\mathrm{G}_{\mathrm{xy}}^{\mathrm{m}}$ - módulo de torção do miolo no plano xy;

$\mathrm{I}^{\mathrm{c}}$ - momento de inércia por unidade de largura das capas, $\mathrm{mm}^{3}$;

$\mathrm{I}^{\mathrm{m}}$ - momento de inércia por unidade de largura do miolo, $\mathrm{mm}^{3}$;

$\eta$ - coeficiente adimensional;

$\mathrm{t}$ - espessura da estrutura do papelão ondulado, mm;

$\mathrm{t}^{\mathrm{c}}$ - espessura do papel-capa, $\mathrm{mm}$;

$\mathrm{t}^{\mathrm{m}}$ - espessura do papel-miolo, $\mathrm{mm}$;

$\mathrm{h}_{\mathrm{c}}$ - altura da onda, $\mathrm{mm}$;

a - ponto médio entre as camadas externas do papelão ondulado, mm;

$v_{x y, y x}^{c}$-coeficiente de Poisson da capa nas direções xy ou yx;

$v^{m}{ }_{x y, y x}$-coeficiente de Poisson do miolo nas direções xy ou yx, e

$\mathrm{p}$ - comprimento da amostra / número de ondas.

\section{RESULTADOS E DISCUSSÃO}

Os dados de resistência à tração e rigidez à flexão (rigidez Taber) para os papéis-capa e miolo, nas direções de fabricação (DF) e transversal (DT), encontram-se na Tabela 3. Os valores de rigidez à flexão refletem com clareza a variação existente entre os papéis-capa e miolo avaliados. Por sua vez, os valores de resistência à tração mostram que a relação observada para os papéis-capa permanece 
crescente com a gramatura, não ocorrendo o mesmo para os papéis-miolo nas gramaturas 120 e 140 para DF. Esse fato pode ser explicado por se utilizar papel reciclado na confecção do miolo, o que não garante a suas características mecânicas.

Na Tabela 4, encontram-se os dados do módulo de elasticidade e coeficiente de Poisson para os papéis-capa e miolo, nas direções de fabricação e transversal, obtidos a partir dos dados de resistência à tração. Os valores de coeficiente de Poisson, para a direção de fabricação, foram obtidos a partir dos dados de percentagem de alongamento na tração na direção transversal, realizados na máquina universal de ensaios e para direção transversal a partir das relações de BAUM \& HABEGER (1980).

TABELA 3. Resistência à tração e rigidez à flexão, dos papéis-capa e miolo.

\begin{tabular}{|c|c|c|c|c|c|c|c|c|}
\hline \multirow{3}{*}{ Papel } & \multicolumn{4}{|c|}{ Resistência à Tração $\left(\mathrm{kN} \mathrm{m}^{-1}\right)$} & \multicolumn{4}{|c|}{ Rigidez Taber $(\mathrm{N} \mathrm{m})$} \\
\hline & \multicolumn{2}{|c|}{ DF } & \multicolumn{2}{|c|}{ DT } & \multicolumn{2}{|c|}{$\mathrm{DF}$} & \multicolumn{2}{|c|}{ DT } \\
\hline & Média & C.V.(\%) & Média & C.V.(\%) & Média & C.V.(\%) & Média & C.V. $(\%)$ \\
\hline C 125 & 11,51 & 6,4 & 4,21 & 4,8 & 1,59 & 9,3 & 0,60 & 10,2 \\
\hline C 140 & 11,90 & 9,9 & 5,28 & 8,3 & 2,33 & 11,5 & 1,06 & 12,4 \\
\hline C 200 & 13,28 & 12,3 & 6,48 & 4,5 & 5,68 & 8,3 & 2,23 & 7,3 \\
\hline M 120 & 6,48 & 2,6 & 2,54 & 5,4 & 1,36 & 8,6 & 0,52 & 11,8 \\
\hline M 140 & 5,98 & 9,1 & 2,96 & 2,6 & 1,47 & 7,4 & 0,57 & 12,4 \\
\hline M 170 & 7,74 & 9,9 & 3,53 & 6,2 & 2,95 & 13,6 & 1,36 & 12,1 \\
\hline
\end{tabular}

C.V. - coeficiente de variação (\%); C - capa seguida pela gramatura; M - miolo seguido pela gramatura.

TABELA 4. Módulo de elasticidade e coeficiente de Poisson dos papéis-capa e miolo.

\begin{tabular}{ccccccccc}
\hline \multirow{2}{*}{ Papel } & \multicolumn{3}{c}{ Módulo de Elasticidade (GPa) } & \multicolumn{3}{c}{ Coeficiente de Poisson $(v)$} \\
\cline { 2 - 9 } & \multicolumn{3}{c}{ DF } & \multicolumn{2}{c}{ DT } & \multicolumn{2}{c}{ DF } & \multicolumn{2}{c}{ DT* } \\
\cline { 2 - 9 } & Média & C.V.(\%) & Média & C.V.(\%) & Média & C.V.(\%) & Média & C.V.(\%) \\
\hline C 125 & 2,12 & 1,47 & 0,43 & 1,83 & 0,55 & 7,64 & 0,14 & 7,46 \\
C 140 & 2,10 & 1,65 & 0,42 & 8,35 & 0,45 & 1,80 & 0,09 & 5,34 \\
C 200 & 1,84 & 1,38 & 0,46 & 1,21 & 0,51 & 1,88 & 0,13 & 1,98 \\
M 120 & 1,82 & 1,02 & 0,40 & 7,46 & 0,56 & 1,29 & 0,12 & 2,31 \\
M 140 & 1,41 & 9,63 & 0,34 & 3,09 & 0,48 & 9,91 & 0,11 & 6,48 \\
M170 & 1,53 & 5,96 & 0,44 & 1,82 & 0,63 & 9,88 & 0,18 & 1,85 \\
\hline
\end{tabular}

*Valores obtidos por meio das relações de BAUM \& HABEGER (1980).

Os valores de módulo de elasticidade, obtidos por meio de ensaios de flexão do papel, estão na Tabela 5 .

TABELA 5. Módulo de elasticidade obtido por meio da Rigidez Taber para os papéis-capa e miolo.

\begin{tabular}{ccccc}
\hline \multirow{2}{*}{ Papel } & \multicolumn{2}{c}{ Módulo de Elasticidade $(\mathrm{GPa})$} & \multicolumn{2}{c}{ Módulo de Elasticidade (GPa) } \\
\cline { 2 - 5 } & DF & C.V.(\%) & DT & C.V.(\%) \\
\hline C 125 & 3,04 & 9,39 & 1,27 & 10,22 \\
C 140 & 3,19 & 11,53 & 1,56 & 12,45 \\
C 200 & 3,02 & 8,32 & 1,20 & 7,31 \\
M 120 & 1,83 & 8,60 & 0,70 & 11,85 \\
M 140 & 1,63 & 7,42 & 0,68 & 12,41 \\
M 170 & 1,58 & 13,63 & 0,79 & 12,16 \\
\hline
\end{tabular}

Na Tabela 6, estão os resultados obtidos na determinação da rigidez à flexão das estruturas de papelão no ensaio de aparelho de quatro pontos. 
TABELA 6. Rigidez das estruturas de papelão ondulado, medida em aparelho de quatro pontos.

\begin{tabular}{ccccc}
\hline \multirow{2}{*}{$\begin{array}{c}\text { Estrutura de } \\
\text { Papelão }\end{array}$} & \multicolumn{4}{c}{ Rigidez à Flexão (N m) } \\
\cline { 2 - 5 } & Média & C.V.(\%) & Média & C.V.(\%) \\
\cline { 2 - 5 } CK45 & 6,16 & 7,99 & 3,42 & 2,81 \\
CK58 & 7,81 & 7,95 & 4,30 & 2,53 \\
BCK85 & 20,50 & 6,24 & 12,79 & 2,73 \\
BCK120 & 40,72 & 3,18 & 19,08 & 3,96 \\
\hline
\end{tabular}

Entre as estruturas de onda $\mathrm{C}$, não se verificaram grandes variações nos valores de rigidez. No entanto, para as estruturas de onda $\mathrm{BC}$, a diferença nos valores de rigidez ficou próxima de $100 \%$ na direção de fabricação e $50 \%$ na direção transversal. A variação nos valores de rigidez reflete a diferença de gramatura dos componentes.

Utilizando a metodologia descrita por CARLSSON et al. (1985), a rigidez foi calculada com os dados de módulo de elasticidade obtidos pelos dois procedimentos de ensaio dos papéis, flexão com o aparelho de Rigidez Taber e tração com os papéis ensaiados na máquina universal. Os resultados da rigidez das estruturas de papelão ondulado encontram-se na Tabela 7.

TABELA 7. Rigidez à flexão de papelão ondulado, calculada pelo procedimento proposto.

\begin{tabular}{ccccc}
\hline \multirow{2}{*}{ Estrutura de Papelão } & \multicolumn{2}{c}{$(1)$ Rigidez $(\mathrm{N} \mathrm{m})$} & \multicolumn{2}{c}{ (2) Rigidez (N m) } \\
\cline { 2 - 5 } & DF & DT & DF & DT \\
\hline CK 45 & 4,7 & 2,3 & 3,3 & 1,1 \\
CK 58 & 5,7 & 3,4 & 3,7 & 1,1 \\
BC 85 & 15,1 & 9,0 & 9,6 & 2,7 \\
BC 120 & 24,8 & 18,2 & 14,3 & 3,2 \\
\hline
\end{tabular}

(1) calculado a partir do Módulo de Elasticidade obtido no ensaio de Rigidez Taber.

(2) calculado a partir do Módulo de Elasticidade obtido no ensaio de tração.

A possibilidade de obter os valores de rigidez para uma estrutura como o papelão ondulado a partir dos módulos de elasticidade dos seus componentes é importante para possíveis avaliações de composições sem a necessidade de ensaiar uma estrutura já fabricada.

Como pode ser observado nos valores da Tabela 7, existe variação expressiva entre os métodos pelos quais se obtêm os módulos de elasticidade, principalmente na direção transversal, e que refletem nos valores de rigidez da estrutura como um todo.

Na Figura 4, os valores de rigidez calculados são mostrados ao lado dos valores obtidos experimentalmente para a direção de fabricação. Na Tabela 8, encontram-se as diferenças entre os valores calculados e os experimentais expressos em percentagem. Observa-se que os valores de rigidez obtidos experimentalmente foram sempre superiores aos valores calculados.

Essa diferença, segundo CARLSSON et al. (1985) e POMMIER et al. (1991), pode ser atribuída a dois fatores conjugados, o processo de fabricação, que confere rigidez maior do que os componentes isoladamente, e a adição da camada adesiva, que não é levada em consideração nos cálculos analíticos. 


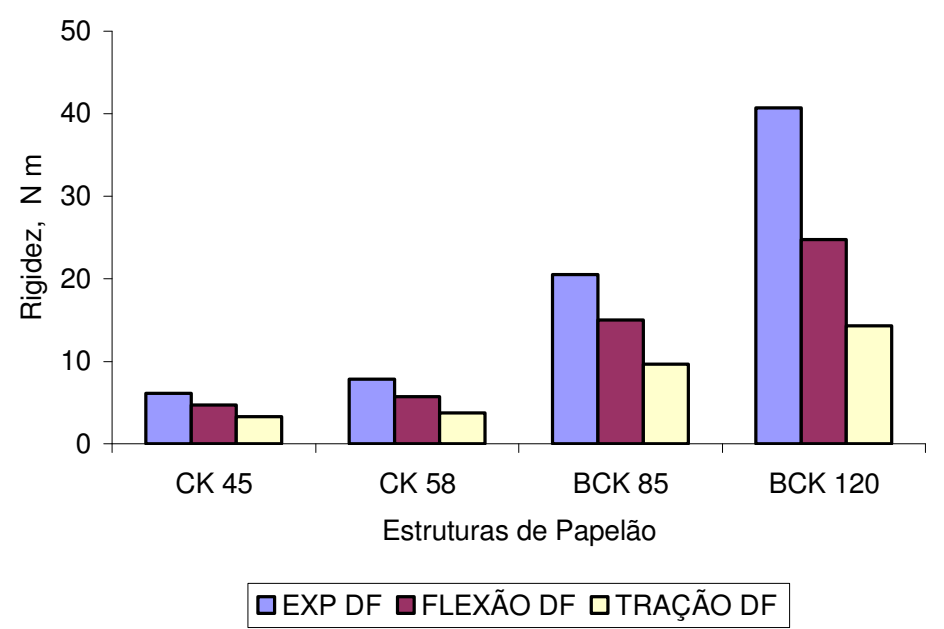

FIGURA 4. Valores de rigidez para as estruturas de papelão ondulado.

TABELA 8. Diferença percentual entre os valores da rigidez à flexão do papelão ondulado, calculado pelos procedimentos propostos, e os obtidos experimentalmente no ensaio de quatro pontos.

\begin{tabular}{lcccc}
\hline \multirow{2}{*}{$\begin{array}{c}\text { Estrutura de } \\
\text { Papelão }\end{array}$} & \multicolumn{4}{c}{ Diferença em Relação ao Ensaio de Quatro Pontos (\%) } \\
\cline { 2 - 5 } & Calculada a partir do ensaio de rigidez Taber & Calculado a partir do ensaio de tração \\
\cline { 2 - 5 } & DF & DT & DF & DT \\
\hline CK 45 & 23,7 & 32,7 & 46,4 & 67,8 \\
CK 58 & 27,0 & 20,9 & 52,6 & 74,4 \\
BC 85 & 26,3 & 29,6 & 53,2 & 78,9 \\
BC 120 & 39,1 & 4,6 & 64,9 & 83,2 \\
\hline Média & 29,0 & 22,0 & 54,3 & 76,1 \\
\hline
\end{tabular}

\section{CONCLUSÕES}

Os valores de rigidez da estrutura do papelão estimados a partir dos ensaios de flexão ou tração dos papéis-capa e miolo, utilizando o módulo de elasticidade calculado, são inferiores aos valores experimentais.

A rigidez estimada com o módulo de elasticidade obtido a partir do ensaio de flexão apresenta valores mais próximos do valor obtido experimentalmente no ensaio de quatro pontos que o estimado pelo método da tração. A diferença média entre os valores da rigidez calculados a partir do ensaio de flexão e os valores experimentais foi de $22 \%$ na direção transversal e de $29 \%$ na direção de fabricação.

\section{AGRADECIMENTOS}

Os autores expressam sua gratidão pelo apoio recebido do $\mathrm{CNPq}$ para a realização desta pesquisa.

\section{REFERÊNCIAS}

BAUM, G.A.; HABEGER, C.C. On-line measurement of paper mechanical properties. Tappi Journal, Atlanta, v.63, n.7, p.63-6, 1980. 
CARLSSON, L.; FELLERS, C.; JONSSON, P. The bending stiffness of corrugated board under special consideration of asymmetrical and multi-ply construction. Das Pappier, Darmstadt, v.39, n.4, p.149-56, 1985.

CHITARRA, M.I.F. Pós-colheita de frutos e hortaliças: fisiologia e manuseio. Lavras: ESAL/FAEPE, 1990. $320 \mathrm{p}$.

MOURAD, A.L.; GARCIA, A.E.; BORDIN, M.R.; ADITO, E.F.G. Embalagens de papel, cartão e papelão ondulado. Campinas: ITAL, 1999. 282 p.

PICHLER, E.F. Embalagem: desenvolvimento, especificação, controle de qualidade e custos. São Paulo: IPT/LEA, 2000. 24 p.

POMMIER, J.C.; POUSTIS, J. New approach for predicting box stacking strength. Revue A.T.I.P., Paris, v.43, n.5, p.217-21, 1989.

POMMIER, J.C.; POUSTIS, J.; FOUCADE, E.; MORLIER, P. Determination of the critical load of a corrugated box subjected to vertical compression by finite elements methods. In: INTERNATIONAL PAPER PHYSICS CONFERENCE, 6., 1991, Kailua Kona. Proceedings ... Kailua Kona: TAPPI, 1991. p.437-47. 